\title{
Mise au point sur les caractéristiques génétiques et les méthodes d'élevage du canard de Barbarie
}

\author{
F.H. RICARD, B. LECLERCQ et H. de CARVILLE \\ I.N.R.A., Station de Recherches avicoles, \\ Centre de Recherches de Tours, \\ Nouzilly, F 37380 Monnaie
}

\section{Résumé}

La production du canard de Barbarie est en augmentation sensible dans de nombreux pays, dont la France. Le présent exposé est une mise au point de nos connaissances concernant quelques caractéristiques génétiques et les méthodes d'élevage du caneton de chair.

En ce qui concerne la génétique, on connait bien les gènes responsables de la coloration du plumage mais assez peu la variabilité des caractères quantitatifs. Les quelques données disponibles permettent cependant de penser qu'une amélioration par la sélection, est possible, comme pour les autres espèces avicoles.

Les principales normes d'élevage ont été résumées ainsi que les besoins alimentaires. Une caractéristique intéressante du caneton de Barbarie est sa faculté de bien ajuster l'ingestion d'énergie. Par rapport à l'aliment consommé, ses besoins en protéines et minéraux paraissent plus faibles que ceux du poulet. Mais, en valeur absolue, du fait que le caneton consomme plus, ses besoins sont comparables.

\section{Introduction}

Les canards de Barbarie appartiennent à l'espèce Cairina moschata L. Par rapport au canard commun Anas platyrhynchos L.), on observe plusieurs séries de différences.

Au point de vue biologique : le canard de Barbarie est un canard percheur d'origine tropicale. C'est un oiseau presque muet. Le mâle présente un développement testiculaire deux fois plus faible que celui du canard commun et son comportement sexuel est moins ardent. De son côté, la femelle entre en ponte plus tard. La durée d'incubation des oufs est plus longue, 35 jours contre 28. C'est par ailleurs un canard plus sensible aux maladies, en particulier aux affections d'origine virale.

Au point de vue anatomique : la principale différence concerne le dimorphisme sexuel de la croissance, beaucoup plus important chez le canard de Barbarie où le poids des femelles dépasse à peine la moitié de celui des mâles. Par ailleurs, la carcasse du canard de Barbarie est connue pour être plus maigre, avec un squelette plus fort et un développement très marqué des masses musculaires pectorales entre 2 et 3 mois 
d'âge. D'autres différences peuvent également être notées : le canard de Barbarie a un croupion plus allongé, des plumes du crâne érectiles, des caroncules, mais pas de plumes caudales chez le mâle.

En France, l'utilisation du canard commun comme animal de chair n'a jamais été très importante, mis à part quelques productions spécialisées comme le canard «nantais» ou le «croisé sauvage». Le canard de Barbarie souffrait au départ d'une productivité moindre. Le fait nouveau est venu de l'amélioration des performances (en particulier de la ponte) et des techniques d'élevage (en particulier la mise au point d'un vaccin contre la maladie de Derzsy). Il en est résulté une intensification de la production, principalement en France et en Italie (Fedeli-AvanzI, 1980). En plus de la production de carcasses à rôtir, on voit maintenant se développer la découpe des carcasses de mâles. Dans ce qui suit, nous nous proposons de faire le point sur ce qui est connu en matière de génétique et de méthodes d'élevage.

\section{Caractéristiques génétiques}

\section{A. Les chromosomes}

Le nombre chromosomique du canard de Barbarie est de $2 \mathrm{~N}=78$, selon l'Atlas chromosomique de BeniRschKe \& Hsu (1973). C'est la quatrième paire (par ordre décroissant de longueur) qui représenterait le chromosome sexuel (X), d'après MotT, LOCKHART \& RIGDON (1968). Selon ces derniers auteurs, les différences morphologiques entre chromosomes analogues des genres Cairina et Anas suffiraient à expliquer la stérilité de l'hybride, le canard mulard.

\section{B. Génétique du plumage}

Un certain nombre de travaux ont été consacrés à l'étude génétique de la coloration du plumage, en Italie par Taibel, aux Etats-Unis par Hollander, en France par Colonna-Cesari. Voici selon ce dernier auteur une liste des gènes connus :

1. Gènes se manifestant seulement sur le duvet

- a : récessif, duvet «atypique » par rapport au type sauvage et plumage noirâtre (TAIBEL, 1956);

- B : duvet atypique (Colonna-Cesari, 1973), dominant par rapport à l'allèle sauvage $\mathrm{b}^{+}$;

- $b^{+}$: récessif, duvet sauvage (COLONNA-CESARI, 1973).

2. Gènes se manifestant à la fois sur le duvet et sur le plumage

- b : récessif, duvet jaune avec queue noire et plumage barré (TAIBEL, 1953, 1954 a) ;

- $\mathrm{p}$ : récessif, duvet jaune et plumage blanc (TAIBEL, 1954 a) ;

— $\mathrm{n}$ : récessif, plumage gris perle (TAIBEL, $1954 \mathrm{~b}$ ); 
- f : récessif, plumage sépia (TAIBEL, 1961);

- 1 : récessif, plumage de couleur lavande (Hollander \& Walther, 1962);

— br : récessif, brun ondulé (Hollander, 1968);

- ch : récessif et lié au sexe, duvet et plumage bruns (Hollander, 1970).

\section{Gènes contrôlant la répartition de la couleur du plumage adulte}

- $\mathrm{d}$ : récessif, taches blanches au cou et au bout des ailes (TAIBEL, 1956) ;

- C : dominant, tête et tiers supérieur du cou blancs (TAibel, 1958).

Nous avons donc, actuellement, une bonne connaissance de la génétique de la couleur du plumage. Le duvet «sauvage» du caneton correspond à un dos noir et à un ventre jaune. Le caneton «atypique " est presque entièrement noir, y compris les pattes. Au locus B, Colonna-CESARI (1973) distingue 3 allèles : $\mathrm{b}^{+}$, correspondant au type «sauvage»; $\mathbf{b}$, correspondant au type «barré »; $\mathbf{B}$, appelé «atypique dominant ». Cette dernière interprétation a été proposée pour expliquer des ségrégations ne correspondant pas aux descriptions de TAIBEL. Le locus $\mathrm{P}(\mathrm{P}=$ couleur possible et $\mathrm{p}=$ blanc récessif) semble analogue au locus $\mathrm{C}$ de la poule. Au locus $\mathrm{N}$, le génotype NN correspond au plumage noir, le génotype $\mathrm{nn}$ au gris perle et l'hétérozygote $\mathrm{Nn}$ à un bleu ardoisé, analogue au «bleu andalou» de la poule domestique.

Du point de vue pratique, la connaissance de ces gènes a permis de créer des variétés homogènes quant à la couleur du plumage, par exemple noires, blanches, barrées, gris-perle.

\section{Relations entre couleur du plumage et caractéristiques zootechniques}

Quelques travaux ont été entrepris pour voir si la couleur du plumage était liée à des caractères intéressants pour les producteurs. En Italie, les variétés noire et blanche ont été comparées par Romboli \& Fedeli-Avanzi (1975) et par Romboli (1976). D'après ces auteurs, la sélection n'aurait porté que sur la couleur du plumage, mais la variété noire a une vitesse de croissance plus grande. Pour des animaux abattus au même poids vif, la variété blanche présente un meilleur rendement à l'abattage, plus de muscles pectoraux et moins de dépôts gras. La viande de la variété noire aurait plus de saveur. Dans un autre travail, Romboli, Finzi \& PITTI (1978) ont comparé les variétés noire et gris-perle : abattus au même poids vif, les canards noirs ont des muscles pectoraux un peu plus développés mais la différence n'est pas significative.

Une expérience de ce type a été entreprise à la Station de Magneraud en 1975 : étaient comparés des canards colorés et blancs en ségrégation dans la même souche, de sexe mâle, et abattus au même âge (12 semaines). Aucune différence n'était significative qu'il s'agisse de poids vif, de la conformation ou de la composition anatomique de la carcasse (RICARD, données non publiées). On peut donc supposer que les gènes de coloration de plumage n'ont pas, par eux-mêmes, d'influence notable sur les caractéristiques de carcasse du canard de Barbarie.

\section{Génétique quantitative}

La variabilité génétique des caractéristiques de productivité du canard de Barbarie a fait l'objet de très peu de publications. En dehors des comparaisons de variétés dont 
nous venons de parler, on peut citer une étude de Leclerce \& DE Carville (1978 c) portant sur 128 canetons d'une même souche : ces auteurs observent des différences d'origine génétique pour l'état d'engraissement et les pertes à l'abattage, mais non pour les muscles pectoraux alors qu'il en existe chez le poulet.

Nous donnons dans le tableau 1 les paramètres statistiques et les valeurs de l'héritabilité observés dans une souche française où un effort était fait pour améliorer à la fois la vitesse de croissance et la ponte. Ces données correspondent à l'ensemble de deux générations de sélection, chacune comprenant 8 lots d'éclosion. Les calculs d'héritabilité ont été faits sur les valeurs transformées en probits, calculés intra-lots d'éclosion selon la technique de Cochez \& Pero (1954). Les contrôles de ponte ont été faits sur des effectifs tronqués, les animaux petits et les familles de faible effectif n'étant pas conservés après 10 semaines d’âge. On constate cependant que la variabilité génétique n'est pas négligeable. C'est le cas notamment pour la précocité sexuelle mesurée par l'âge au premier œuf, ce qui laisse penser qu'on peut améliorer ce caractère par la sélection. L'héritabilité du poids vif est assez élevée et celle de l'intensité de ponte relativement faible, comme chez la poule domestique.

\section{TABleau 1}

Paramètres de la croissance et de la ponte observés dans une souche française de canards de Barbarie au cours des années 1975 et 1976

(données observées en élevage privé).

Statistical parameters and heritability values for growth rate, age at first egg and laying intensity in a French strain of muscovy ducks (1975 and 1976).

\begin{tabular}{|c|c|c|c|c|}
\hline & \multicolumn{2}{|c|}{$\begin{array}{l}\text { Poids à } 10 \text { semaines }(\mathrm{g}) \\
\text { Weight at } 10 \text { weeks }\end{array}$} & \multirow{2}{*}{$\begin{array}{l}\text { Age au } \\
1^{\text {er }} \text { cuf } \\
\text { (jours) } \\
\text { Age } \\
\text { at first egg } \\
\text { (d) }\end{array}$} & \multirow{2}{*}{$\begin{array}{l}\text { Intensité } \\
\text { de ponte } \\
\text { (p. 100) } \\
\text { Laying rate } \\
(\text { p. 100) }\end{array}$} \\
\hline & $\begin{array}{l}\text { Mâles } \\
\text { Males }\end{array}$ & $\begin{array}{l}\text { Femelles } \\
\text { Females }\end{array}$ & & \\
\hline $\begin{array}{l}\text { Effectifs contrôlés : } \\
\text { Controled } \\
\text { Nombre de pères } \ldots \ldots \ldots \ldots \ldots \ldots \\
\text { Number of sires }\end{array}$ & 160 & 160 & 140 & 140 \\
\hline $\begin{array}{l}\text { Nombre de mères } \ldots \ldots \ldots \ldots \ldots \\
\text { Number of clams }\end{array}$ & 626 & 626 & 321 & 32.1 \\
\hline $\begin{array}{l}\text { Nombre d'eafants } \ldots \ldots \ldots \ldots \\
\text { Number of offsprings }\end{array}$ & 4453 & 4442 & 2897 & 2883 \\
\hline $\begin{array}{l}\text { Moyerne - Mean ............... } \\
\text { Ecart-type - Standard deviation }\end{array}$ & $\begin{array}{r}2896 \\
296 \\
\end{array}$ & $\begin{array}{r}1852 \\
148 \\
\end{array}$ & $\begin{array}{r}231 \\
17\end{array}$ & $\begin{array}{l}65,9 \\
16,4\end{array}$ \\
\hline $\begin{array}{l}\text { Héritalibités - Heritability : } \\
\text { Composante père } \ldots \ldots \ldots \ldots \ldots \ldots \\
\text { Sire component }\end{array}$ & 0,24 & 0,47 & 0,55 & $0,2: 1$ \\
\hline $\begin{array}{l}\text { Composante mère } \ldots \ldots \ldots \ldots \cdots \\
\text { Dam component }\end{array}$ & 0,62 & 0,49 & 0,43 & 0,11 \\
\hline
\end{tabular}




\section{Méthodes d'élevage}

Ce chapitre emprunte de nombreux renseignements à l'ouvrage de H. DE CARville \& A. De Croutte, Le Canard (1978).

\section{A. Le bâtiment d'élevage}

L'un des intérêts de l'élevage du caneton de Barbarie est qưil peut se faire dans des poulaillers classiques sur le plan de l'isolation et de la ventilation. Du fait de ses origines, laccès à un bassin n'est pas utile. Par contre, certaines particularités physiologiques doivent être prises en compte. Daprès MonTlaur (1974), le canard ne disposerait à la naissance que de reins embryonnaires non immédiatement fonctionnels. Il est donc sensible à une déshydratation et assure plus difficilement sa régulation thermique, ce qui implique de veiller à son abreuvement au cours des premiers jours et d'utiliser un mode de chauffage régulier et non desséchant. Par ailleurs, son besoin en oxygène est supérieur à celui des autres oiseaux, ce qui demande une ventilation correcte du local d’élevage.

Une première technique consiste à élever les canetons au sol, sur litière de paille ou de copeaux de bois. Mais, les fientes de canard sont beaucoup plus aqueuses que celles du poulet et après 5 semaines d’âge, lentretien devient astreignant et coûteux car il faut prévoir des apports journaliers de litière fraîche.

Une autre technique consiste à élever les animaux sur caillebotis de bois ou sur grillage. Le caillebotis consiste en des lattes de 20 à $25 \mathrm{~mm}$ de côté avec écartement de $15 \mathrm{~mm}$ entre deux lattes. Des cages munies de grillage sont utilisées dans notre Station car c'est une technique bien adaptée à l'expérimentation et qui assure une simplification du travail. Nous utilisons des mailles de $20 \times 20 \mathrm{~mm}$, avec feuilles de papier durant la première semaine. Dans d'autres élevages, on utilise des mailles rectangulaires de $25 \times 12,5 \mathrm{~mm}$.

Quel que soit l'aménagement intérieur, un bâtiment obscur et une claustration complète permettent une meilleure maîtrise de l'élevage. Les normes, connues avec moins de précision que pour le poulet, sont résumées ci-dessous.

\section{Densité}

4 mâles ou 6 femelles par mètre carré sur litière, 6 mâles ou 8 femelles sur caillebotis ou sur grillage. Dans certains élevages, ces densités sont largement dépassées.

\section{Température}

$$
\begin{array}{lr}
1^{\mathrm{r}} & \text { semaine : } 35^{\circ} \mathrm{C} \text { sous éleveuse, } 18 \text { à } 20^{\circ} \mathrm{C} \text { en ambiance } \\
2^{\mathrm{e}} & \text { semaine : } 30-32^{\circ} \mathrm{C} \text { sous éleveuse, } 18 \text { à } 20^{\circ} \mathrm{C} \text { en ambiance } \\
3^{\circ} & \text { semaine : } 28-30^{\circ} \mathrm{C} \text { sous éleveuse, } 16 \text { à } 18{ }^{\circ} \mathrm{C} \text { en ambiance } \\
4^{\prime \prime} & \text { semaine : } 23-26^{\circ} \mathrm{C} \text { sous éleveuse, } 15 \text { à } 18{ }^{\circ} \mathrm{C} \text { en ambiance } \\
5^{\circ} & \text { semaine : } 20-211^{\circ} \mathrm{C} \text { sous éleveuse, } 15 \text { à } 18^{\circ} \mathrm{C} \text { en ambiance. }
\end{array}
$$




\section{Ventilation}

De $4 \mathrm{~m}^{3}$ /heure l'hiver à $10 \mathrm{~m}^{3} /$ heure l'été par kilogramme de poids vif.

\section{Eclairement}

Il est bon de prévoir un éclairage continu pendant la première semaine puis une réduction progressive d'une heure par jour jusqu'à $10 \mathrm{~h}$ de lumière par $24 \mathrm{~h}$. L'intensité sera de 50 lux la première semaine, puis 5 à 10 lux avec utilisation éventuelle de la lumière rouge.

\section{Matériel}

Il est important d'assurer un abreuvement facile et abondant ainsi que l'accès simultané de tous les animaux à la mangeoire. On peut recommander :

- au démarrage pour 250 sujets : 5 abreuvoirs siphoïdes de deux litres et 4 mètres linéaires de mangeoire;

- après deux semaines et pour 250 sujets : un abreuvoir linéaire de deux mètres et 8 mètres de mangeoire.

\section{B. Problème du picage}

C'est l'une des difficuités de l'élevage du caneton de Barbarie. Il se manifeste d'habitude vers l'âge de 3 à 4 semaines au niveau de la queue et du dos, vers 6 à 7 semaines pour les ailes. On peut diminuer les risques en distribuant un aliment équilibré, en limitant l'intensité lumineuse, en utilisant de la lumière rouge, en effectuant un débecquage préventif dès l'âge de 3 semaines. On peut aussi dégriffer les animaux de façon à éviter des blessures qui diminuent considérablement la qualité commerciale des carcasses.

\section{L'alimentation du jeune canard}

Plusieurs séries d'expériences ont été entreprises à la Station de Recherches avicoles ces dernières années, en vue de définir les besoins du caneton de Barbarie et de voir en quoi ils diffèrent des autres espèces de volailles, en particulier du poulet.

\section{Taux énergétique}

Dans la gamme des conditions habituelles de formulation, le taux énergétique de l'aliment n'influence pas la croissance du caneton de Barbarie (LECLERcQ \& DE CARVILle, 1976 b ; LeclercQ, 1981). Par contre, l'indice de consommation est meilleur avec des taux élevés comme le montre le tableau 2. Des taux élevés ont tendance à donner des animaux plus gras : 4,5 $\mathrm{g}$ de lipides supplémentaires, par $\mathrm{kg}$ de poids vif, pour une élévation de $100 \mathrm{Kcal}$ d'énergie métabolisable par $\mathrm{kg}$ d'aliment, ce qui ne représente qu'une incidence modérée.

\section{Rationnement}

Quelques essais de rationnement en finition, en vue de réduire l'état d'engraissement, se sont avérés d'un intérêt limité. En effet, la diminution des dépôts adipeux s'accompagne d'un léger ralentissement de la croissance, d'un indice de consommation souvent moins bon, et les risques de picage augmentent (LECLERCQ \& DE CARville, 1978 a). 


\section{TABleau 2}

Influence du taux énergétique sur la croissance et l'indice de consommation du caneton de Barbarie.

Influence of energy level on 10 wk live weight and 3-10 wk feed conversion.

(from LeCLERCQ, 1981).

\begin{tabular}{|c|c|c|c|}
\hline $\begin{array}{l}\text { Taux énergétique } 11 \text { ) } \\
\text { Energy level }\end{array}$ & 2660 & 2880 & 3100 \\
\hline $\begin{array}{l}\text { Poids vif à } 10 \text { semaines }(g) \ldots \ldots \ldots \ldots \ldots \ldots \\
\text { Live weight at } 10 \text { weeks }\end{array}$ & 3324 & 3403 & 3395 \\
\hline $\begin{array}{l}\text { Indice de consommation entre } 3 \text { et } 10 \text { semaines. } \\
\text { Feed conversion between } 3 \text { and } 10 \text { weeks }\end{array}$ & 2,92 & 2,66 & 2,50 \\
\hline
\end{tabular}

(1) En Kcal d'énergie métabolisable par $\mathrm{kg}$ d'aliment - In Kcal metabolisable energy/ $\mathrm{kg}$ food.

Un rationnement en début de croissance peut être utilisé en vue de réduire les accidents attribués à une surcharge des reins et du foie. L'indice de consommation est amélioré de 5 à 10 p. 100 sans perte de croissance ultérieure, car les canards rattrapent en finition le retard pris au démarrage (Tremolieres, 1976).

\section{Taux protéique}

Les études concernant le besoin en protéines ont montré que le caneton de Barbarie pouvait se contenter dès la naissance d'un taux modéré de matières azotées totales. Ainsi, dans le tableau 3 , on peut voir que la meilleure croissance à 3 semaines est obtenue avec un taux de matières azotées totales de l'ordre de $190 \mathrm{~g} / \mathrm{kg}$ d'aliment pour un taux énergétique de $3000 \mathrm{Kcal}$ d'énergie métabolisable par $\mathrm{kg}$ d'aliment. Entre 4 et 6 ou 8 semaines, le besoin n'excède pas $150 \mathrm{~g} / \mathrm{kg}$ et il s'abaisse vers une valeur de 120 à 130 au-delà (LeClercQ \& DE CARville, 1975 et 1976 a).

\section{TABLEAU 3}

Influence du taux azoté sur le poids vif à 3 semaines d'âge, pour des aliments contenant $2950 \mathrm{Kcal}$ d'EM/ $\mathrm{kg}$.

Influence of protein level on 3 wh live weight, for a same energy level.

(from Leclerce \& De Carville, 1976 b)

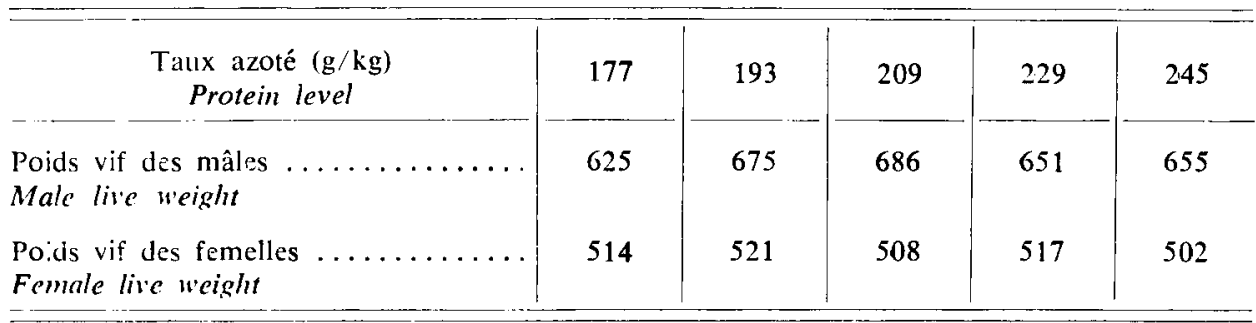


Au-delà de 3 semaines d'âge, les variations possibles des conditions de milieu font qu'il est préférable d'exprimer l'apport de protéines en quantité ingérée pour une croissance donnée pendant une période donnée (LeCLERCQ \& DE CARVILLE, 1978 b et 1981). Un exemple de ce calcul est donné dans le tableau 4.

\section{Besoin en acides aminés essentiels}

Exprimé en quantité ingérée, le besoin en lysine peut être estimé à $24 \mathrm{~g}$ pour un gain de poids de $1,5 \mathrm{~kg}$ entre 3 et 6 semaines et de $35 \mathrm{~g}$ entre 6 et 10 semaines (LeClercQ \& DE Carville, 1979 a). Une supplémentation en lysine de synthèse permet de diminuer l'apport en protéines totales (cf. tabl. 4).

\section{TABleau 4}

Besoins en protéines du caneton de Barbarie mâle, exprimé en grammes pour un croît de $1,5 \mathrm{~kg}$.

Protein needs of muscovy ducklings, in grams for 1500 grams weight gain, without or with added lysine in the feed.

\begin{tabular}{|c|c|c|}
\hline $\begin{array}{l}\text { Age du caneton } \\
\text { Duckling age }\end{array}$ & $\begin{array}{l}3 \text { à } 6 \text { sem. } \\
3 \text { to } 6 \text { weeks }\end{array}$ & $\begin{array}{l}6 \text { à } 10 \text { sem. } \\
6 \text { to } 10 \text { weeks }\end{array}$ \\
\hline $\begin{array}{l}\text { Aliment sans lysine de synthèse } \ldots \ldots \ldots \ldots \ldots \ldots \\
\text { Feed without lysine }\end{array}$ & 590 & 860 \\
\hline $\begin{array}{l}\text { Avec supplémentation en lysine de synthèse } \ldots \ldots \\
\text { With lysine }\end{array}$ & 480 & 700 \\
\hline
\end{tabular}

Le besoin en acides aminés soufrés est plus difficile à établir car la carence n'est pas facile à réaliser, en particulier en finition. Une addition de $1 \mathrm{~g}$ de méthionine de synthèse par $\mathrm{kg}$ d'aliment donne quand même de meilleurs résultats. Mais on constate qu'un régime contenant $2900 \mathrm{Kcal}$ d'énergie métabolisable par $\mathrm{kg}$ et $5 \mathrm{~g}$ dacides aminés soufrés, c'est-à-dire un aliment maïs-soja classique, permet d'obtenir des croissances très convenables (LeCLerce \& DE CARville, 1977).

Un essai récent du même type, mais avec un régime de base à $3000 \mathrm{Kcal}$ d'EM/ $\mathrm{kg}$ et $139 \mathrm{~g} / \mathrm{kg}$ de protéines brutes, a donné les résultats indiqués au tableau 5. Une supplémentation en méthionine de $1 \mathrm{~g} / \mathrm{kg}$ améliore significativement la croissance. L'indice de consommation et l'état d'engraissement sont abaissés, mais de façon non significative.

\section{Besoin en minéraux}

En ce qui concerne le besoin en phosphore, il est préférable, là aussi, de le fixer en valeur absolue en fonction du gain de poids et de l'âge. Daprès LeclercQ \& DE Carville (1979 b), ce besoin est de 4 grammes de phosphore assimilable entre 0 et 3 semaines, de $6,5 \mathrm{~g}$ entre 3 et 6 semaines, de $11 \mathrm{~g}$ entre 6 et 10 semaines pour des gains de poids de l'ordre de $600 \mathrm{~g}, 1350 \mathrm{~g}$ et $1500 \mathrm{~g}$ respectivement, chez le caneton mâle. 


\section{TABleau 5}

Influence d'une supplémentation en méthionine pour un aliment à $3000 \mathrm{Kcal}$ d'EM par $\mathrm{kg}$ d'aliment (données non publiées).

Influence of added methionine in a feed with $3000 \mathrm{Kcal} \mathrm{EM/kg}$ (unpublished data).

\begin{tabular}{|c|c|c|c|}
\hline $\begin{array}{l}\text { Teneur du régime en acides aminés soufrés }(\mathrm{g} / \mathrm{kg}) \ldots \ldots \ldots \\
\text { Diet level in sulphur amino acids }\end{array}$ & 5,0 & 6,0 & 7,0 \\
\hline $\begin{array}{l}\text { Méthionine ajoutée }(\mathrm{g} / \mathrm{kg}) \ldots \ldots \ldots \ldots \ldots \ldots \ldots \ldots \\
\text { Added methionine }\end{array}$ & 0 & 1,0 & 2,0 \\
\hline $\begin{array}{l}\text { Poids vif à } 6 \text { semaines }(g) \ldots \ldots \ldots \ldots \ldots \ldots \ldots \ldots \ldots \ldots \\
\text { Live weight at } 6 \text { weeks }\end{array}$ & 2252 & 2285 & 2281 \\
\hline $\begin{array}{l}\text { Gain de poids } 6-11 \text { semaines }(\mathrm{g})(1) \ldots \ldots \ldots \ldots \ldots \ldots \ldots \\
\text { Weight gain : } 6-11 \text { weeks }\end{array}$ & 1338 & 1427 & 1409 \\
\hline $\begin{array}{l}\text { Indice de consommation } 6-11 \text { semaines } \ldots \ldots \ldots \ldots \ldots \ldots \\
\text { Feed conversion } 6-11 \text { weeks }\end{array}$ & 4,51 & 4,30 & 4,39 \\
\hline $\begin{array}{l}\text { Gras abdominal (à } 11 \text { sem.) (en } \mathrm{g} / 100 \mathrm{~g} \text { poids vif) } \ldots \ldots \ldots \\
\text { Abdominal fat (at } 11 \text { weeks) (g/100 g live weight) }\end{array}$ & 2,17 & 2,07 & 1,97 \\
\hline
\end{tabular}

\section{TABLEAU 6}

Extrait des recommandations de la Station de Recherches avicoles de l'I.N.R.A. concernant l'alimentation du caneton de Barbarie (plaquette I.N.R.A., 1979).

I.N.R.A., Poultry Research Station recommendations for the main feed characteristics, given for two energy levels and three ages $(0-3,3-6$ and over 6 weeks $)$.

\begin{tabular}{|c|c|c|c|c|c|c|}
\hline Taux énergétique & \multicolumn{3}{|c|}{$2600 \mathrm{Kcal} \mathrm{EM} / \mathrm{kg}$} & \multicolumn{3}{|c|}{$3000 \mathrm{Kcal} \mathrm{EM} / \mathrm{kg}$} \\
\hline $\begin{array}{c}\text { Type d'aliment } \\
\text { Feed type }\end{array}$ & $\begin{array}{l}\text { Démar- } \\
\text { rage } \\
0-3 \text { sem. } \\
\text { Starter }\end{array}$ & $\begin{array}{l}\text { Crois- } \\
\text { sance } \\
\text { 3-6 sem. } \\
\text { Growth }\end{array}$ & $\begin{array}{l}\text { Finition } \\
>6 \text { sem. } \\
\text { Finishing }\end{array}$ & $\begin{array}{l}\text { Démar- } \\
\text { rage } \\
0-3 \text { sem. } \\
\text { Starter }\end{array}$ & $\begin{array}{l}\text { Crois- } \\
\text { sance } \\
\text { 3-6 sem. } \\
\text { Growth }\end{array}$ & $\begin{array}{l}\text { Finition } \\
>6 \text { sem. } \\
\text { Finishing }\end{array}$ \\
\hline \multicolumn{7}{|l|}{$\begin{array}{l}\text { En } \mathrm{g} / \mathrm{kg} \text { d'aliment : } \\
\mathrm{g} / \mathrm{kg} \text { feed }\end{array}$} \\
\hline $\begin{array}{l}\text { Matières azotées totales } \\
\text { Total crude protein }\end{array}$ & 165 & 139 & 120 & 190 & 160 & 145 \\
\hline Lysine $\ldots \ldots \ldots \ldots$ & 8,3 & 6,6 & 6,0 & 9,6 & 7,6 & 7,0 \\
\hline Méthionine $\ldots \ldots \ldots$ & 3,6 & 2,9 & 2,2 & 4,1 & 3,3 & 2,6 \\
\hline Acides aminés soufrés & 7,0 & 5,7 & 4,7 & 8,0 & 6,5 & 5,4 \\
\hline Calcium $\ldots \ldots \ldots \ldots$ & 8,0 & 7,0 & 6,0 & 9,0 & 8,0 & 7,0 \\
\hline Phosphore assimilable . & 3,6 & 3,0 & 2,2 & 4,0 & 3,5 & 2,5 \\
\hline \multicolumn{7}{|c|}{$\begin{array}{l}\text { Nota : Ces normes correspondent à des poids vifs de l'ordre de } 0,65 \mathrm{~kg} \text { à } 3 \text { semaines, } 2 \mathrm{~kg} \mathrm{à} \\
6 \text { semaines et } 3,8 \mathrm{~kg} \text { à } 12 \text { semaines pour les mâles; } 0,6 \mathrm{~kg} \text { à } 3 \text { semaines, } 1,6 \mathrm{~kg} \text { à } 6 \text { semaines } \\
\text { et } 2,2 \mathrm{~kg} \text { à } 10 \text { semaines pour les femelles - These recommendations correspond to live weights of } \\
0.65 \mathrm{~kg} \text { at } 3 \text { weeks, } 2 \mathrm{~kg} \text { at } 6 \text { weeks and } 3.8 \mathrm{~kg} \text { at } 12 \text { weeks for males; } 0.6 \mathrm{~kg} \text { at } 3 \text { weeks, } \\
1.6 \mathrm{~kg} \text { at } 6 \text { weeks and } 2.2 \mathrm{~kg} \text { at } 10 \text { weeks for females. }\end{array}$} \\
\hline
\end{tabular}


Pour les autres éléments minéraux, les études manquent mais sont moins justifiées car les supplémentations sont le plus souvent excessives quelle que soit l'espèce considérée.

\section{Comparaison caneton-poulet}

Contrairement au poulet, le caneton de Barbarie présente un bon ajustement de l'ingestion d'énergie métabolisable pour une large gamme de concentrations énergétiques (Blum \& LeclercQ, 1979 ; LeclercQ, 1981).

On pourrait penser que le caneton est moins exigeant en protéines que le poulet. En réalité, on obtient des chiffres similaires quand les besoins sont exprimés en valeur absolue. Ce qui distingue le plus les deux espèces, c'est leur ingestion de constituants énergétiques, c'est-à-dire d'aliment, la consommation du caneton étant supérieure à celle du poulet. On comprend donc que par rapport à l'aliment, le besoin du caneton paraisse plus faible. Le même raisonnement est valable pour les autres éléments de la ration, en particulier les minéraux.

En guise de synthèse des travaux ci-dessus, nous donnons dans le tableau 6 un extrait des recommandations de la Station de Recherches avicoles établies pour deux taux énergétiques, 2600 et $3000 \mathrm{Kcal}$ d'énergie métabolisable par $\mathrm{kg}$ d'aliment (I.N.R.A., 1979).

Accepté pour publication en mars 1983.

\section{Remerciements}

La plupart des chiffres présentés dans les tableaux ont été obtenus sur cles animaux fournis par la Société Grimaud Frères (49450 Roussay, France). Nous remercions vivement MM. Grimaud dont l'esprit de coopération a grandement facilité les travaux entrepris à l'I.N.R.A. sur le canard de Barbarie.

\section{Summary}

Some genetic characteristics and rearing techniques of muscovy ducklings (Cairina moschata $L$.)

Muscovy duckling production is increasing in a number of countries, especially in France. This paper reviews the present knowledge about some genetic traits, as well as rearing techniques with emphasis on nutritional characteristics.

On the genetic level, plumage colour genes are now well known, but very few experiments have been published dealing with variability of quantitative characters. Heritability values for growth rate and laying parameters are given in table 1 . They are in favour of a significant improvement by breeding means.

The main technical parameters are summarized. On the nutritional side, results obtained at the Nouzilly Poultry Research Station are summarized : energy level (tabl. 2), restricted feeding, total protein and essential amino acid needs (tabl. 3, 4 and 5), mineral requirements. An interesting feature is the ability of muscovy ducklings to correctly adjust their energy input. When compared to food intake, protein and mineral needs seem to be lower for muscovies than for chickens. In fact, muscovy ducklings eat more and, in absolute value, their needs are comparable to those of chickens. As a synthesis, table 6 give the main recommendations for feeding muscovy ducklings until 12 weeks of age. 


\section{Références bibliographiques}

Benirschke K., Hsu T.C., 1973. Cairina moschata. In : "Chromosome Atlas» : Fish, amphibians, reptiles and birds, 2, folio Av-23, Springer-Verlag, Berlin.

Blum J.C., Leclerce B., 1979. Comparaison of species. Poultry int., 18, 80-84.

de Carville H., de Croutte A., 1978. Le canard, Editions Vigot, Paris, 15-103.

Cochez L.P., Pero R., 1954. Sélection des caractères économiques. Transformation des mesures brutes en écarts réduits pour atténuer l'incidence des variations de milieu. Proc. 10th World's Poultry Cong., Edinburgh, 16-19.

Colonna-Cesari X., 1973. La sélection du carnard de Barbarie. Document ronéoté, cours approfondi d'Amélioration Génétique des Animaux Domestiques, 10 pages, I.N.A., Paris.

Fedeli-Avanzi C., 1980. Muscovy duck : a breed which will develop. Poultry int., 19. $14-20$.

Hollander W.F., 1968. Brown-rippled, a recessive mutant in the muscovy duck. J. Hered., 59, 309-311.

Holdander W.F., 1970. Sex-linked chocolate coloration in the muscovy duck. Poultry Sci., 49, 594-596.

Hollander W.F., Walther P.L., 1962. Recessive lavender in the muscovy duck. J. Hered., 53, 81-83.

I.N.R.A., Station de Recherches avicoles, 1979. L'alimentation des volailles : Le caneton de chair. $2^{\circ}$ édition, S.E.I., C.N.R.A., Versailles, 12 pages.

Leclerce B., 1981. L'alimentation du canard de Barbarie est bien spécifique. Le Courrier Avicole, 803, 19-22.

Leclerce B., de Carville H., 1975. Besoin en protéines du caneton de Barbarie entre les âges de 4 et 11 semaines. Ann. Zootech., 24, 217-227.

Leclerce B., De Carville H., 1976 a. L'alimentation azotée du caneton de Barbarie : étude du besoin du caneton mâle entre les âges de 4 et 12 semaines. Ann. Zootech., 25, 189-197.

Leclerce B., de Carville H., 1976 b. Influence of protein and energy contents of the diet on the growth of young muscovy ducklings. Arch. Geflügelk., 40, 117-119.

Leclerce B., de Carville H., 1977. On the sulfur amino acid requirement of muscovy ducklings. Arch. Gefluigelk., 41, 270-272.

Leclerce B., de Carville H., 1978 a. Intérêt du rationnement du caneton mâle de Barbarie entre les âges de 8 et 12 semaines. Ann. Zootech., 27, 1-7.

Leclerce B., de Carville H., 1978 b. L'alimentation azotée du caneton de Barbarie : possibilités de réduction du taux protidique de l'aliment au cours de la période de finition. Ann. Zootech., 27, 169-174.

Leclerce B., de Carville H., $1978 \mathrm{c}$. Variabilité de quelques composants corporels chez le canard de Barbarie. Proc. 16th World's Poultry Congr., Rio de Janeiro, 12, 2034-2035.

Leclerce B., de Carville H., 1979 a. The lysine requirement of muscovy ducklings determined with corn-sunflower meal diets. Arch. Geflügelk, 43, 69-72.

Leclerce B., de Carville H., 1979 b. Le besoin en phosphore du caneton de Barbarie. Ann. Zootech., 28, 101-107.

Leclerce B., de Carville H., 1981. Protein requirement of muscovy ducklings fed corn soybean meal diets supplemented with lysine and methionine. Arch. Geflügelk., 45, 92-95.

Montlaur P., 1974. Répercussions pathologiques de certains aspects de l'anatomie et de la physiologie de l'oie et du canard. Document ronéoté, U.C.A.A.B. Château-Thierry, 24 pages.

Mott C.L., Lockhart L.H., Rigdon R.H., 1968. Chromosomes of the sterile hybrid duck. Cytogenetics, 7, 403-412. 
Romboli I., 1976. Caratteristiche produttive in due varieta di anatra muschiata. Simp. Nutr. aliment. Specie minore, Roma 1975, Cons. Naz. Ric., 211-224.

Romboli I., Fedeli-Avanzi C., 1975. Plumage colour factors and meat quality in muscovy duck. Proc. 2nd Europ. Symp. Poultry Meat, Oosterbeek, paper 30, 5 pages, Editor : Dutch Branch W.P.S.A., Beekbergen.

Romboli I., Finzi A., Pitti A., 1978. Valutazione delle carcasse e delle carni dell' anatra muschiata. Avicoltura, 47, 31-35.

Taibel A.M., 1953. Nuova varieta di colore del plumaggio dell'anatra muschiata, Cairina moschata domestica L., ottenuta per mutazione. Ann. sper. Agric. N.S., 7, 1823-1828.

TAibel A.M., 1954 a. Comportamento genetico del nuovo carattere «Bar» dell'anatra Muschiata. Ann. sper. Agric. N.S., 8, 1005-1023.

Taibel A.M., 1954 b. Il plumagio "grigio-ardesia-azzurrastro» dell'anatra Muschiata e suo valore genetico. Ann. sper. Agric. N.S., 8, 1795-1801.

Taibel A.M., 1956. Nuove mutazioni, causa di nuove varieta, nel corredo cromosomico della anatra Muschiata. Ann. sper. Agric. N.S., 10, 2143-2155.

TAIBEL A.M., 1958. Un nuovo "fattore di repartizione» causa della "pezzatura bianca al copa e al terzo superiore del collo», nel corredo cromosomico dell'anatra Muschiata. Ann. sper. Agric. N.S., 12, 537-553.

TAiBel A.M., 1961. Due nuove verieta di colore nel plumaggio dell'anatra Muschiata sorte per mutazione. Boll. Zool., 28, 561-568.

Tremolieres M.E., 1976. L'alimentation pratique du canard de Barbarie. Session «canard à rôtir $»$, Cholet, juin 1976. Document U.C.A.A.B., Château-Thierry, 16 pages +18 tableaux et figures. 